\title{
What is the job satisfaction and active participation of medical staff in public hospital reform: a study in Hubei province of China
}

Pengqian Fang ${ }^{1 * \dagger}$, Zhenni Luo $^{2 \dagger}$ and Zi Fang ${ }^{3}$

\begin{abstract}
Background: In China, public hospital reform has been underway for almost 5 years, and 311 pilot county hospitals are the current focus. This study aimed to assess the job satisfaction and active participation of medical staff in the reform. A total of 2268 medical staff members in pilot and non-pilot county hospitals in Hubei, China, were surveyed.

Methods: Questionnaires were used to collect data. The Pearson chi-square statistical method was used to assess the differences between pilot and non-pilot county hospitals and identify the factors related to job satisfaction as well as the understanding and perception of the reform. Binary logistic regression was performed to determine the significant factors that influence the job satisfaction of medical staff in pilot county hospitals.

Results: Medical staff members in pilot county hospitals expressed higher satisfaction on current working situation, performance appraisal system, concern showed by leaders, hospital management, and compensation packages $(P<0.05)$. They were exposed to work-related stress at a higher extent $(P<0.05)$ and half of them worked overtime. Within pilot county hospitals, less than half of the medical staff members were satisfied with current job and they have evidently less satisfaction on compensation packages and learning and training opportunities. The working hours and work stress were negatively related to the job satisfaction $(P<0.05)$. Satisfaction on the performance appraisal system, hospital management, compensation packages, and learning and training opportunities were positively related to job satisfaction $(P<0.05)$. Medical staff in pilot county hospitals exhibited better understanding of and more positive attitude towards the reform $(P<0.05)$.

Conclusions: Pilot county hospitals have implemented some measures through the reform, but there still are deficiencies. The government officials and hospital administrators should pay attention to influencing factors of job satisfaction and focus on the reasonable demands of medical staff. In addition, the medical staff in pilot county hospitals exhibited a better understanding of the public hospital reform programme and showed more firm confidence, but there still were some medical staff members who hold negative attitude. The publicity and education of the public hospital reform still need improvement.
\end{abstract}

Keywords: Medical staff, China, Public hospital reform, Working situation, Satisfaction, Understanding, Perception

\footnotetext{
* Correspondence: pfang@mails.tjmu.edu.cn

${ }^{\dagger}$ Equal contributors

'School of Health and Medicine Management, Tongji Medical College, Huazhong University of Science and Technology, 13 Hangkong Road, Qiaokou District, Wuhan 430030, China

Full list of author information is available at the end of the article
} 


\section{Background}

In October 2008, a draft of Healthcare Reform Plan was published by the Chinese government, and comments were solicited from the whole society. In this way, the general public was able to participate in policy-making in China, and this initiative is unprecedented [1,2]. In March 2009, China's Healthcare Reform Plan was formally released to reduce the residents' economic burden for medical services, mitigate the difficulties in affordability and accessibility of medical service, and provide safe, effective, convenient, inexpensive health services for universal coverage. The short-term goals of the health-care reform comprise five key programmes: expedite the establishment of a basic medical security system, initiate a national basic drugs system, improve the primary healthcare service system, promote the gradual equalization of basic public health services, and implement a pilot programme of public hospital reform.

The pilot reform of public hospitals includes the following contents: improve health service systems and establish medical cooperative activities between urban and rural, substantially increase financial investment into hospitals, remove the provision of commercial hospital services, reform the compensation system of public hospitals, restructure the management systems of public hospitals, enhance income distribution and incentive programmes, and encourage social organizations to open hospitals [3]. Former premier Wen Jiabao considered public hospital reform as one of the most important and challenging tasks in the new health reform. Public hospitals are the principal medical and health service institutions in China. As such, these institutions are essential for public welfare in medical and health services. Therefore, public hospital reform is an important factor for the realization of health-care reform in China.

The pilot programme of county hospital reform is the threshold and emphasis of public hospital reform, and county hospitals are currently in focus. China's Ministry of Health selected 311 county hospitals on a national scale as pilot hospitals, and 20 of the 311 are located in Hubei province. These 311 pilot county hospitals must take the lead to carry out the measures and contents of public hospitals reform mentioned above, while the rest of the county hospitals could remain original.

County hospitals are considered as flagships of threetiered rural health-care systems and the main provider of health services; these services include treatment and emergency services, disease prevention, vaccination, health education, maternal and child health-care services, and reproductive services for rural residents [4], who account for more than $50 \%$ of the total Chinese population, and some urban residents. In China, a county hospital is generally equipped with 300 to 500 beds and approximately 400 to 600 medical staff members, providing health services for approximately 0.5 million individuals in an area of 2000 to $4000 \mathrm{~km}^{2}$.

Medical staff is the direct provider of hospital services and key element in the development of health services. The work performance, attitude, and commitment of medical staff directly influence the outcomes of health service delivery, such as medical safety, service quality, doctor-patient relationship, patient satisfaction, and hospital management, particularly the operating efficiency and effectiveness of a hospital. Medical staff members in pilot county hospitals are also responsible for the implementation and promotion of the reform policies as well as the generation of support for the reform.

To our knowledge, studies on the working situation, satisfaction, and attitude towards the reform of medical staff in pilot county hospitals in China in this specific period are few [5,6], let alone on comparison between pilot and non-pilot county hospitals. The purposes of this study are to describe and compare the working situation, satisfaction, and attitude towards the reform of medical staff in pilot county hospitals with those of medical staff in non-pilot county hospital in Hubei province and to analyse and determine the influencing factors of medical staff in pilot county hospitals. We aim to improve job satisfaction and arouse active participation in public hospital reform, to provide high-quality medical services to residents.

\section{Methods}

\section{Study population}

County hospitals were selected by sampling at several stages. Considering that Hubei province is a large area in central China, we initially selected seven cities in east, south, west, north, northwest, northeast, and central Hubei according to geographical locations and economic development levels. They are Wuhan city, Jingzhou city, Yichang city, Xiangyang city, Shiyan city, Suizhou city, and Jingmen city, respectively. There are 20 pilot county hospitals in Hubei province. Two pilot counties and one non-pilot county were then randomly selected from each of the seven cities. One county hospital was finally chosen from each of the 21 counties. Thus, this study comprised 21 county hospitals, including 14 pilot county hospitals and 7 non-pilot county hospitals.

In each selected county hospital, 110 medical staff members, including doctors, nurses, and medical technicians (personnel in pharmacy, clinical laboratory, and radiology department) were randomly chosen and considered as subjects of the study. A total of 2310 medical staff members from 1531 in pilot county hospitals and 737 in non-pilot county hospitals were investigated and 2268 valid answer sheets were returned, resulting in a response rate of $98.18 \%$. This study was approved by the Ethics Committee of Tongji Medical College, Huazhong University of Science 
and Technology (IRB No: FWA00007304). The medical staff was aware of this study and willing to participate. The privacy of the investigated medical staff was strictly protected by filling in the questionnaires anonymously.

\section{Questionnaire}

Adapting the Minnesota Satisfaction Questionnaire based on the actual situation of medical staff members of county hospitals in China, this study formulated a questionnaire and its index system through questionnaire investigation, literature survey, and Delphi expert consultation. The questionnaire consisted of three parts: Part 1, sociodemographic information; Part 2, working situation and satisfaction; and Part 3, understanding and perception of the reform. Through reliability statistics, Cronbach's alpha is 0.913 .

Part 1 included sociodemographic information of gender, age, educational background, position, professional title, and years in professional working experience.

In Part 2, working situation included two aspects: the number of hours spent at work every day and the work stress one felt. For the number of hours, six options were provided from " $\leq 8 \mathrm{~h}$ " to " $\geq 12 \mathrm{~h}$ ". For work stress, five options were provided from "no pressure" to "extreme pressure". In Part 2, satisfaction included six aspects: current job, performance appraisal system, concern showed by leaders, hospital management, compensation packages, and learning and training opportunities. For these aspects, five options (numbered from 1 to 5 ) were provided to express satisfaction degree: 1, very satisfied; 2, satisfied; 3, moderate/acceptable; 4, dissatisfied; and 5 , very dissatisfied.

In Part 3, investigation on the understanding and perception of the reform included the following: understanding of the specific contents of the public hospital reform programme, perceived level of reform promotion, changes experienced before and after the reform introduction in 2008, and the perception of the effect of the public hospital reform.

\section{Statistical analysis}

EpiData3.1 was used to establish a database, and double machine inputting method was used to enter the collected data into the computer. PASW Statistics 18.0 was used to perform statistical data analysis. The sociodemographic factors of the investigated medical staff were summarized using a descriptive statistical analysis method. The Pearson chi-square statistical method was used to assess the differences between medical staff in pilot county hospitals and medical staff in non-pilot county hospitals in working situation, satisfaction on job and wok-related factors, and the understanding and perception of the reform. The Pearson chi-square statistical method was also used to analyse the factors influencing job satisfaction and the understanding and perception of the reform in pilot county hospitals. Binary logistic regression was then performed to determine significant factors influencing job satisfaction in pilot county hospitals. The dependent variable (the outcome of interest) in logistic regression included the following: 1 "satisfied" (very satisfied, satisfied, moderate) and 0 "otherwise" (dissatisfied, very dissatisfied). We substituted the variables with statistical significance determined from the Pearson chi-square statistical test into the binary logistic regression model for calculation $(P<0.05)$. Odds ratio (OR) was reported at a $95 \%$ confidence interval (CI) where appropriate. All of the tests were conducted at $5 \%$ significance level.

\section{Results}

Sociodemographic characteristics of the investigated medical staff in pilot and non-pilot county hospitals

The investigated medical staff members were mainly comprised of females (66.58\%). The largest proportion of age of the medical staff ranged from 25 to 34 (38.27\%). The largest proportion of educational background was bachelor's degree (48.19\%), and the largest proportion of professional title was middle title (43.83\%; Table 1). In this study, different professional titles showed various professional skill levels of the medical staff members. In China, a medical staff member needs to undergo and pass a special qualification examination and assessment to achieve his (or her) professional title corresponding to a particular professional skill level.

\section{Working situation, job satisfaction, and satisfaction on work-related factors of medical staff in pilot and non-pilot county hospitals}

In pilot county hospitals, $51.86 \%$ of the medical staff members work for more than $8 \mathrm{~h}$ a day and $55.39 \%$ are exposed to considerable pressure or extreme pressure. The percentages of medical staff members in pilot county hospitals dissatisfied or very dissatisfied with current job, performance appraisal system, concern showed by leaders, hospital management, compensation packages, and learning and training opportunities were 17.37\%, 15.55\%, $10.65 \%, 6.53 \%, 35.41 \%$, and $47.42 \%$, respectively.

Through Pearson chi-square statistical analysis, the results showed that medical staff in pilot and non-pilot county hospitals exhibited significant differences in work stress and satisfaction on the following aspects: current job, performance appraisal system, concern showed by leaders, hospital management, and compensation packages $(P<0.001$; Table 2$)$.

\section{Analysis and determining of the significant factors influencing the job satisfaction of medical staff in pilot county hospitals}

In pilot county hospitals, only $45.72 \%$ of medical staff expressed that they were very satisfied or satisfied with 
Table 1 Sociodemographic characteristics of medical staff in pilot and non-pilot county hospitals

\begin{tabular}{|c|c|c|c|c|c|c|}
\hline \multirow[t]{2}{*}{ Characteristic } & \multicolumn{2}{|l|}{ Pilot county hospitals } & \multicolumn{2}{|c|}{ Non-pilot county hospitals } & \multicolumn{2}{|l|}{ Total } \\
\hline & Medical staff $(n=1531)$ & Percentage (\%) & Medical staff $(n=737)$ & Percentage (\%) & $n$ & $\%$ \\
\hline \multicolumn{7}{|l|}{ Gender } \\
\hline Male & 552 & 36.05 & 206 & 27.95 & 758 & 33.42 \\
\hline Female & 979 & 63.95 & 531 & 72.05 & 1510 & 66.58 \\
\hline \multicolumn{7}{|l|}{ Age } \\
\hline 24 and below & 150 & 9.80 & 63 & 8.55 & 213 & 9.39 \\
\hline $25-34$ & 527 & 34.42 & 341 & 46.27 & 868 & 38.27 \\
\hline $35-44$ & 559 & 36.51 & 212 & 28.77 & 771 & 33.99 \\
\hline $45-54$ & 268 & 17.50 & 107 & 14.52 & 375 & 16.53 \\
\hline 55 and above & 27 & 1.76 & 14 & 1.90 & 41 & 1.81 \\
\hline \multicolumn{7}{|l|}{ Educational background } \\
\hline Technical secondary school and below & 188 & 12.28 & 48 & 6.51 & 236 & 10.41 \\
\hline Junior college & 547 & 35.73 & 216 & 29.31 & 763 & 33.64 \\
\hline Bachelor's degree & 737 & 48.14 & 356 & 48.30 & 1093 & 48.19 \\
\hline Master's degree or above & 59 & 3.85 & 117 & 15.88 & 176 & 7.76 \\
\hline \multicolumn{7}{|l|}{ Position } \\
\hline Doctor & 599 & 39.12 & 280 & 37.99 & 879 & 38.76 \\
\hline Nurse & 661 & 43.17 & 357 & 48.44 & 1018 & 44.89 \\
\hline Medical technician & 271 & 17.70 & 100 & 13.57 & 371 & 16.36 \\
\hline \multicolumn{7}{|l|}{ Professional title } \\
\hline No title & 24 & 1.57 & 11 & 1.49 & 35 & 1.54 \\
\hline Junior title & 513 & 33.51 & 333 & 45.18 & 846 & 37.30 \\
\hline Middle title & 729 & 47.62 & 265 & 35.96 & 994 & 43.83 \\
\hline Senior title & 265 & 17.31 & 128 & 17.37 & 393 & 17.33 \\
\hline \multicolumn{7}{|l|}{ Years in professional working experience } \\
\hline $1-5$ years & 288 & 18.81 & 196 & 26.59 & 484 & 21.34 \\
\hline $6-10$ years & 214 & 13.98 & 141 & 19.13 & 355 & 15.65 \\
\hline $11-15$ years & 227 & 14.83 & 81 & 10.99 & 308 & 13.58 \\
\hline $16-20$ years & 307 & 20.05 & 135 & 18.32 & 442 & 19.49 \\
\hline 20 years and above & 495 & 32.33 & 184 & 24.97 & 679 & 29.94 \\
\hline \multicolumn{7}{|l|}{ City } \\
\hline Wuhan city & 220 & 14.37 & 108 & 14.65 & 328 & 14.46 \\
\hline Jingzhou city & 218 & 14.24 & 105 & 14.25 & 323 & 14.24 \\
\hline Yichang city & 215 & 14.04 & 98 & 13.30 & 313 & 13.80 \\
\hline Xiangyang city & 220 & 14.37 & 104 & 14.11 & 324 & 14.29 \\
\hline Shiyan city & 219 & 14.30 & 108 & 14.65 & 327 & 14.42 \\
\hline Suizhou city & 219 & 14.30 & 105 & 14.25 & 324 & 14.29 \\
\hline Jingmen city & 220 & 14.37 & 109 & 14.79 & 329 & 14.51 \\
\hline
\end{tabular}

current job. About $36.90 \%$ of medical staff felt moderate, and $17.37 \%$ felt dissatisfied or very dissatisfied with current job. Medical staff was evidently less satisfied with compensation packages (18.55\% only) and learning and training opportunities $(18.29 \%$ only) than other work-related aspects (Table 3 ).
Through Pearson chi-square statistical analysis, the results showed that factors related to the job satisfaction of medical staff in pilot county hospitals included age, educational background, position, professional title, years in professional working experience, the number of hours spent at work, work stress, and satisfaction on the 
Table 2 Working situation, job satisfaction, and satisfaction on work-related factors of medical staff in pilot and non-pilot county hospitals

\begin{tabular}{|c|c|c|c|c|c|c|c|c|}
\hline \multirow[t]{2}{*}{ Working situation and satisfaction } & \multicolumn{2}{|c|}{$\begin{array}{l}\text { Pilot county } \\
\text { hospitals }(n=1531)\end{array}$} & \multicolumn{2}{|c|}{$\begin{array}{l}\text { Non-pilot county } \\
\text { hospitals }(n=737)\end{array}$} & \multicolumn{2}{|l|}{ Total } & \multirow[t]{2}{*}{$x^{2}$} & \multirow[t]{2}{*}{$P$} \\
\hline & $\bar{n}$ & $\%$ & $n$ & $\%$ & $n$ & $\%$ & & \\
\hline \multicolumn{9}{|c|}{ The number of hours spent at work everyday } \\
\hline $8 \mathrm{~h}$ and below & 737 & 48.14 & 394 & 53.46 & 1131 & 49.87 & & \\
\hline $9 \mathrm{~h}$ & 407 & 26.58 & 171 & 23.20 & 578 & 25.49 & & \\
\hline $10 \mathrm{~h}$ & 219 & 14.30 & 103 & 13.98 & 322 & 14.20 & & \\
\hline $11 \mathrm{~h}$ & 46 & 3.00 & 14 & 1.90 & 60 & 2.65 & & \\
\hline $12 \mathrm{~h}$ & 37 & 2.42 & 16 & 2.17 & 53 & 2.34 & & \\
\hline $12 \mathrm{~h}$ and above & 85 & 5.55 & 39 & 5.29 & 124 & 5.47 & 7.582 & 0.181 \\
\hline \multicolumn{9}{|l|}{ The degree of work stress } \\
\hline No pressure & 31 & 2.02 & 168 & 22.80 & 199 & 8.77 & & \\
\hline Slight pressure & 411 & 26.85 & 157 & 21.30 & 568 & 25.04 & & \\
\hline Moderate pressure & 241 & 15.74 & 150 & 20.35 & 391 & 17.24 & & \\
\hline Considerable pressure & 682 & 44.55 & 198 & 26.87 & 880 & 38.80 & & \\
\hline Extreme pressure & 166 & 10.84 & 64 & 8.68 & 230 & 10.14 & 299.217 & $<0.001$ \\
\hline \multicolumn{9}{|l|}{ Satisfaction on current job } \\
\hline Very satisfied & 136 & 8.88 & 38 & 5.16 & 174 & 7.67 & & \\
\hline Satisfied & 564 & 36.84 & 205 & 27.82 & 769 & 33.91 & & \\
\hline Moderate & 565 & 36.90 & 313 & 42.47 & 878 & 38.71 & & \\
\hline Dissatisfied & 239 & 15.61 & 158 & 21.44 & 397 & 17.50 & & \\
\hline Very dissatisfied & 27 & 1.76 & 23 & 3.12 & 50 & 2.20 & 38.744 & $<0.001$ \\
\hline \multicolumn{9}{|c|}{ Satisfaction on performance appraisal system } \\
\hline Very satisfied & 34 & 2.22 & 12 & 1.63 & 46 & 2.03 & & \\
\hline Satisfied & 475 & 31.03 & 101 & 13.70 & 576 & 25.40 & & \\
\hline Moderate & 784 & 51.21 & 404 & 54.82 & 1188 & 52.38 & & \\
\hline Dissatisfied & 197 & 12.87 & 180 & 24.42 & 377 & 16.62 & & \\
\hline Very dissatisfied & 41 & 2.68 & 40 & 5.43 & 81 & 3.57 & 111.369 & $<0.001$ \\
\hline \multicolumn{9}{|c|}{ Satisfaction on concern showed by leaders } \\
\hline Very satisfied & 76 & 4.96 & 11 & 1.49 & 87 & 3.84 & & \\
\hline Satisfied & 517 & 33.77 & 134 & 18.18 & 651 & 28.70 & & \\
\hline Moderate & 775 & 50.62 & 433 & 58.75 & 1208 & 53.26 & & \\
\hline Dissatisfied & 133 & 8.69 & 117 & 15.88 & 250 & 11.02 & & \\
\hline Very dissatisfied & 30 & 1.96 & 42 & 5.70 & 72 & 3.17 & 109.148 & $<0.001$ \\
\hline \multicolumn{9}{|l|}{ Satisfaction on hospital management } \\
\hline Very satisfied & 77 & 5.03 & 13 & 1.76 & 90 & 3.97 & & \\
\hline Satisfied & 608 & 39.71 & 162 & 21.98 & 770 & 33.95 & & \\
\hline Moderate & 746 & 48.73 & 417 & 56.58 & 1163 & 51.28 & & \\
\hline Dissatisfied & 83 & 5.42 & 118 & 16.01 & 201 & 8.86 & & \\
\hline Very dissatisfied & 17 & 1.11 & 27 & 3.66 & 44 & 1.94 & 145.094 & $<0.001$ \\
\hline \multicolumn{9}{|l|}{ Satisfaction on compensation packages } \\
\hline Very satisfied & 20 & 1.31 & 8 & 1.09 & 28 & 1.23 & & \\
\hline Satisfied & 264 & 17.24 & 40 & 5.43 & 304 & 13.40 & & \\
\hline Moderate & 705 & 46.05 & 227 & 30.80 & 932 & 41.09 & & \\
\hline Dissatisfied & 460 & 30.05 & 336 & 45.59 & 796 & 35.10 & & \\
\hline
\end{tabular}


Table 2 Working situation, job satisfaction, and satisfaction on work-related factors of medical staff in pilot and non-pilot county hospitals (Continued)

\begin{tabular}{|c|c|c|c|c|c|c|c|c|}
\hline Very dissatisfied & 82 & 5.36 & 126 & 17.10 & 208 & 9.17 & 189.192 & $<0.001$ \\
\hline \multicolumn{9}{|c|}{ Satisfaction on learning and training opportunities } \\
\hline Very satisfied & 34 & 2.22 & 11 & 1.49 & 45 & 1.98 & & \\
\hline Satisfied & 246 & 16.07 & 101 & 13.70 & 347 & 15.30 & & \\
\hline Moderate & 525 & 34.29 & 252 & 34.19 & 777 & 34.26 & & \\
\hline Dissatisfied & 481 & 31.42 & 247 & 33.51 & 728 & 32.10 & & \\
\hline Very dissatisfied & 245 & 16.00 & 126 & 17.10 & 371 & 16.36 & 4.193 & 0.38 \\
\hline
\end{tabular}

following aspects: performance appraisal system, concern showed by leaders, hospital management, compensation packages, and learning and training opportunities $(P<0.05$; Table 3$)$.

To further determine the significant factors influencing the job satisfaction of medical staff in pilot county hospitals, we substituted the variables with statistical significance from the previous Pearson chi-square statistical analysis into the binary logistic regression model for calculation. The dependent variable $Y$ in logistic regression included the following: 1 "satisfied" (very satisfied, satisfied, moderate) and 0 "dissatisfied" (dissatisfied, very dissatisfied). The independent variable $X 1$ (age) included five grades from younger to older. The independent variable X2 (position) included "doctor", "nurse", and "medical technician". The independent variable X3 (professional title) included four grades from lower to higher. The independent variable $X 4$ (years in professional working experience) included five grades from shorter to longer. The independent variable $X 5$ (educational background) included four grades from lower to higher. The independent variable $X 6$ (the number of hours spent at work every day) included four grades from less to greater. The independent variable $X 7$ (work stress) included two grades: 1 "no pressure or moderate pressure" (no pressure, slight pressure, moderate pressure) and 2 "great pressure" (considerable pressure, extreme pressure). The independent variables $X 8$ (satisfaction on performance appraisal system), $X 9$ (satisfaction on concern showed by leaders), $X 10$ (satisfaction on hospital management), X11 (satisfaction on compensation packages), and $X 12$ (satisfaction on learning and training opportunities) all included two grades: 1 "satisfied" (very satisfied, satisfied, moderate) and 2 "dissatisfied" (dissatisfied, very dissatisfied).

The results of the binary logistic regression analysis showed that the factors significantly influencing the job satisfaction of medical staff in pilot county hospitals included the number of hours spent at work, work stress, satisfaction on performance appraisal system, satisfaction on hospital management, satisfaction on compensation packages, and satisfaction on learning and training opportunities $(P<0.05$; Table 4$)$. In particular, the job satisfaction probability of the medical staff who worked for 9,10 , and $11 \mathrm{~h}$ every day were 0.483 times $(\mathrm{OR}=0.483,95 \% \mathrm{CI}=$ $0.327-0.713, P<0.001), 0.364$ times $(\mathrm{OR}=0.364,95 \% \mathrm{CI}=$ $0.230-0.576, P<0.001)$, and 0.339 times $(\mathrm{OR}=0.339,95 \%$ $\mathrm{CI}=0.202-0.568, P<0.001)$ lower than that of the medical staff who worked for a maximum of $8 \mathrm{~h}$, respectively. The job satisfaction probability of the medical staff who felt no pressure or moderate pressure was 2.384 times higher $(\mathrm{OR}=2.384,95 \% \mathrm{CI}=1.666-3.411, P<0.001)$ than that of the medical staff who felt great work pressure. These results showed that working hours and work pressure were negatively related to job satisfaction in pilot county hospitals. The job satisfaction probability of the medical staff who were satisfied with the performance appraisal system was 2.243 times higher than that of the medical staff who were dissatisfied $(\mathrm{OR}=2.243,95 \% \mathrm{CI}=1.504-$ $3.345, P<0.001)$. The job satisfaction probability of the medical staff who were satisfied with hospital management was 2.043 times higher than that of the medical staff who were dissatisfied $(\mathrm{OR}=2.043,95 \% \mathrm{CI}=1.196-3.490, P=$ 0.009 ). The job satisfaction probability of the medical staff who were satisfied with compensation packages was 3.298 times higher than that of the medical staff who were dissatisfied $(\mathrm{OR}=3.298,95 \% \mathrm{CI}=2.410-4.513, P<0.001)$. The job satisfaction probability of the medical staff who were satisfied with learning and training opportunities was 1.442 times higher than that of the medical staff who were dissatisfied $(\mathrm{OR}=1.442,95 \% \mathrm{CI}=1.050-$ $1.980, P=0.024)$. These results showed that satisfaction on performance appraisal system, hospital management, compensation packages, and learning and training opportunities were positively related to job satisfaction in county hospitals.

\section{Understanding and perception of the public hospital reform of medical staff in pilot and non-pilot county hospitals}

In pilot county hospitals, only $35.92 \%$ of the investigated medical staff members expressed that they knew a lot or fully knew of the specific contents of the public hospital reform programme and policy. Only $50.29 \%$ of the investigated medical staff members expressed that they obviously 
Table 3 Assessment on the factors related to job satisfaction of medical staff in pilot county hospitals

\begin{tabular}{|c|c|c|c|c|c|c|c|c|c|c|c|c|}
\hline \multirow{3}{*}{$\begin{array}{l}\text { Sociodemographic characteristics, working } \\
\text { situation, and satisfaction on work-related } \\
\text { factors }\end{array}$} & \multicolumn{10}{|c|}{ Job satisfaction of medical staff in pilot county hospitals $(n=1531)$} & \multirow[t]{3}{*}{$x^{2}$} & \multirow[t]{3}{*}{$P$} \\
\hline & \multicolumn{2}{|c|}{ Very satisfied } & \multicolumn{2}{|c|}{ Satisfied } & \multicolumn{2}{|c|}{ Moderate } & \multicolumn{2}{|c|}{ Dissatisfied } & \multicolumn{2}{|c|}{ Very dissatisfied } & & \\
\hline & $n$ & $\%$ & $n$ & $\%$ & $n$ & $\%$ & $n$ & $\%$ & $n$ & $\%$ & & \\
\hline \multicolumn{13}{|l|}{ Gender } \\
\hline Male & 44 & 7.97 & 210 & 38.04 & 196 & 35.51 & 88 & 15.94 & 14 & 2.54 & & \\
\hline Female & 92 & 9.40 & 354 & 36.16 & 369 & 37.69 & 151 & 15.42 & 13 & 1.33 & 4.588 & 0.332 \\
\hline \multicolumn{13}{|l|}{ Age } \\
\hline 24 and below & 14 & 9.33 & 48 & 32.00 & 65 & 43.33 & 21 & 14.00 & 2 & 1.33 & & \\
\hline $25-34$ & 45 & 8.54 & 169 & 32.07 & 216 & 40.99 & 84 & 15.94 & 13 & 2.47 & & \\
\hline $35-44$ & 40 & 7.16 & 211 & 37.75 & 207 & 37.03 & 93 & 16.64 & 8 & 1.43 & & \\
\hline $45-54$ & 30 & 11.19 & 123 & 45.90 & 75 & 27.99 & 37 & 13.81 & 3 & 1.12 & & \\
\hline 55 and above & 7 & 25.93 & 13 & 48.15 & 2 & 7.41 & 4 & 14.81 & 1 & 3.70 & 44.291 & $<0.001$ \\
\hline \multicolumn{13}{|l|}{ Educational background } \\
\hline Technical secondary school and below & 32 & 17.02 & 64 & 34.04 & 61 & 32.45 & 28 & 14.89 & 3 & 1.60 & & \\
\hline Junior college & 49 & 8.96 & 210 & 38.39 & 209 & 38.21 & 75 & 13.71 & 4 & 0.73 & & \\
\hline Bachelor's degree & 54 & 7.33 & 278 & 37.72 & 265 & 35.96 & 122 & 16.55 & 18 & 2.44 & & \\
\hline Master's degree or above & 1 & 1.69 & 12 & 20.34 & 30 & 50.85 & 14 & 23.73 & 2 & 3.39 & 39.668 & $<0.001$ \\
\hline \multicolumn{13}{|l|}{ Position } \\
\hline Doctor & 40 & 6.68 & 208 & 34.72 & 215 & 35.89 & 116 & 19.37 & 20 & 3.34 & & \\
\hline Nurse & 58 & 8.77 & 242 & 36.61 & 257 & 38.88 & 98 & 14.83 & 6 & 0.91 & & \\
\hline Medical technician & 38 & 14.02 & 114 & 42.07 & 93 & 34.32 & 25 & 9.23 & 1 & 0.37 & 42.361 & $<0.001$ \\
\hline \multicolumn{13}{|l|}{ Professional title } \\
\hline No title & 3 & 12.50 & 7 & 29.17 & 12 & 50.00 & 2 & 8.33 & 0 & 0.00 & & \\
\hline Junior title & 40 & 7.80 & 159 & 30.99 & 216 & 42.11 & 86 & 16.76 & 12 & 2.34 & & \\
\hline Middle title & 68 & 9.33 & 288 & 39.51 & 256 & 35.12 & 107 & 14.68 & 10 & 1.37 & & \\
\hline Senior title & 25 & 9.43 & 110 & 41.51 & 81 & 30.57 & 44 & 16.60 & 5 & 1.89 & 21.666 & 0.041 \\
\hline \multicolumn{13}{|l|}{ Years in professional working experience } \\
\hline $1-5$ years & 26 & 9.03 & 81 & 28.13 & 123 & 42.71 & 52 & 18.06 & 6 & 2.08 & & \\
\hline $6-10$ years & 17 & 7.94 & 64 & 29.91 & 91 & 42.52 & 35 & 16.36 & 7 & 3.27 & & \\
\hline $11-15$ years & 13 & 5.73 & 93 & 40.97 & 89 & 39.21 & 30 & 13.22 & 2 & 0.88 & & \\
\hline $16-20$ years & 27 & 8.79 & 111 & 36.16 & 113 & 36.81 & 50 & 16.29 & 6 & 1.95 & & \\
\hline 20 years and above & 53 & 10.71 & 215 & 43.43 & 149 & 30.10 & 72 & 14.55 & 6 & 1.21 & 38.586 & 0.001 \\
\hline \multicolumn{13}{|l|}{ City } \\
\hline Wuhan city & 17 & 7.73 & 87 & 39.55 & 81 & 36.82 & 33 & 15.00 & 2 & 0.91 & & \\
\hline Jingzhou city & 12 & 5.50 & 82 & 37.61 & 84 & 38.53 & 37 & 16.97 & 3 & 1.38 & & \\
\hline Yichang city & 20 & 9.30 & 78 & 36.28 & 73 & 33.95 & 40 & 18.60 & 4 & 1.86 & & \\
\hline Xiangyang city & 22 & 10.00 & 72 & 32.73 & 89 & 40.45 & 29 & 13.18 & 8 & 3.64 & & \\
\hline Shiyan city & 23 & 10.50 & 77 & 35.16 & 78 & 35.62 & 34 & 15.53 & 7 & 3.20 & & \\
\hline Suizhou city & 16 & 7.31 & 78 & 35.62 & 86 & 39.27 & 38 & 17.35 & 1 & 0.46 & & \\
\hline Jingmen city & 26 & 11.82 & 90 & 40.91 & 74 & 33.64 & 28 & 12.73 & 2 & 0.91 & 27.146 & 0.298 \\
\hline \multicolumn{13}{|l|}{ The number of hours spent at work everyday } \\
\hline $8 \mathrm{~h}$ and below & 97 & 13.16 & 308 & 41.79 & 260 & 35.28 & 66 & 8.96 & 6 & 0.81 & & \\
\hline $9 \mathrm{~h}$ & 26 & 6.39 & 148 & 36.36 & 155 & 38.08 & 74 & 18.18 & 4 & 0.98 & & \\
\hline $10 \mathrm{~h}$ & 9 & 4.11 & 65 & 29.68 & 84 & 38.36 & 56 & 25.57 & 5 & 2.28 & & \\
\hline $11 \mathrm{~h}$ & 1 & 2.17 & 13 & 28.26 & 23 & 50.00 & 7 & 15.22 & 2 & 4.35 & & \\
\hline
\end{tabular}


Table 3 Assessment on the factors related to job satisfaction of medical staff in pilot county hospitals (Continued)

\begin{tabular}{|c|c|c|c|c|c|c|c|c|c|c|c|c|}
\hline $12 \mathrm{~h}$ & 1 & 2.70 & 8 & 21.62 & 17 & 45.95 & 9 & 24.32 & 2 & 5.41 & & \\
\hline $12 \mathrm{~h}$ and above & 2 & 2.35 & 22 & 25.88 & 26 & 30.59 & 27 & 31.76 & 8 & 9.41 & 141.127 & $<0.001$ \\
\hline \multicolumn{13}{|l|}{ The degree of work stress } \\
\hline No pressure & 14 & 45.16 & 10 & 32.26 & 5 & 16.13 & 2 & 6.45 & 0 & 0.00 & & \\
\hline Slight pressure & 56 & 13.63 & 194 & 47.20 & 129 & 31.39 & 27 & 6.57 & 5 & 1.22 & & \\
\hline Moderate pressure & 29 & 12.03 & 91 & 37.76 & 96 & 39.83 & 25 & 10.37 & 0 & 0.00 & & \\
\hline Considerable pressure & 34 & 4.99 & 225 & 32.99 & 287 & 42.08 & 126 & 18.48 & 10 & 1.47 & & \\
\hline Extreme pressure & 3 & 1.81 & 44 & 26.51 & 48 & 28.92 & 59 & 35.54 & 12 & 7.23 & 222.417 & $<0.001$ \\
\hline \multicolumn{13}{|c|}{ Satisfaction on performance appraisal system } \\
\hline Very satisfied & 22 & 64.71 & 4 & 11.76 & 6 & 17.65 & 1 & 2.94 & 1 & 2.94 & & \\
\hline Satisfied & 80 & 16.84 & 257 & 54.11 & 107 & 22.53 & 30 & 6.32 & 1 & 0.21 & & \\
\hline Moderate & 28 & 3.57 & 258 & 32.91 & 364 & 46.43 & 126 & 16.07 & 8 & 1.02 & & \\
\hline Dissatisfied & 5 & 2.54 & 42 & 21.32 & 74 & 37.56 & 67 & 34.01 & 9 & 4.57 & & \\
\hline Very dissatisfied & 1 & 2.44 & 3 & 7.32 & 14 & 34.15 & 15 & 36.59 & 8 & 19.51 & 483.816 & $<0.001$ \\
\hline \multicolumn{13}{|c|}{ Satisfaction on concern showed by leaders } \\
\hline Very satisfied & 35 & 46.05 & 30 & 39.47 & 9 & 11.84 & 2 & 2.63 & 0 & 0.00 & & \\
\hline Satisfied & 79 & 15.28 & 258 & 49.90 & 132 & 25.53 & 47 & 9.09 & 1 & 0.19 & & \\
\hline Moderate & 19 & 2.45 & 132 & 17.03 & 363 & 46.84 & 135 & 17.42 & 12 & 1.55 & & \\
\hline Dissatisfied & 2 & 1.50 & 47 & 35.34 & 56 & 42.11 & 42 & 31.58 & 8 & 6.02 & & \\
\hline Very dissatisfied & 1 & 3.33 & 1 & 3.33 & 5 & 16.67 & 13 & 43.33 & 6 & 20.00 & 427.387 & $<0.001$ \\
\hline \multicolumn{13}{|c|}{ Satisfaction on hospital management } \\
\hline Very satisfied & 38 & 49.35 & 30 & 38.96 & 9 & 11.69 & 0 & 0.00 & 0 & 0.00 & & \\
\hline Satisfied & 79 & 12.99 & 311 & 51.15 & 165 & 27.14 & 50 & 8.22 & 3 & 0.49 & & \\
\hline Moderate & 17 & 2.28 & 204 & 27.35 & 364 & 48.79 & 148 & 19.84 & 13 & 1.74 & & \\
\hline Dissatisfied & 1 & 1.20 & 18 & 21.69 & 25 & 30.12 & 31 & 37.35 & 8 & 9.64 & & \\
\hline Very dissatisfied & 1 & 5.88 & 1 & 5.88 & 2 & 11.76 & 10 & 58.82 & 3 & 17.65 & 466.78 & $<0.001$ \\
\hline \multicolumn{13}{|c|}{ Satisfaction on compensation packages } \\
\hline Very satisfied & 11 & 55.00 & 7 & 35.00 & 1 & 5.00 & 0 & 0.00 & 1 & 5.00 & & \\
\hline Satisfied & 60 & 22.73 & 139 & 52.65 & 57 & 21.59 & 8 & 3.03 & 0 & 0.00 & & \\
\hline Moderate & 39 & 5.53 & 286 & 40.57 & 294 & 41.70 & 81 & 11.49 & 5 & 0.71 & & \\
\hline Dissatisfied & 24 & 5.22 & 123 & 26.74 & 186 & 40.43 & 116 & 25.22 & 11 & 2.39 & & \\
\hline Very dissatisfied & 2 & 2.44 & 9 & 10.98 & 27 & 32.93 & 34 & 41.46 & 10 & 12.20 & 363.14 & $<0.001$ \\
\hline \multicolumn{13}{|c|}{ Satisfaction on learning and training opportunities } \\
\hline Very satisfied & 11 & 32.35 & 11 & 32.35 & 10 & 29.41 & 1 & 2.94 & 1 & 2.94 & & \\
\hline Satisfied & 42 & 17.07 & 114 & 46.34 & 69 & 28.05 & 20 & 8.13 & 1 & 0.41 & & \\
\hline Moderate & 48 & 9.14 & 218 & 41.52 & 178 & 33.90 & 76 & 14.48 & 5 & 0.95 & & \\
\hline Dissatisfied & 22 & 4.57 & 147 & 30.56 & 204 & 42.41 & 94 & 19.54 & 14 & 2.91 & & \\
\hline Very dissatisfied & 13 & 5.31 & 74 & 30.20 & 104 & 42.45 & 48 & 19.59 & 6 & 2.45 & 112.753 & $<0.001$ \\
\hline
\end{tabular}

perceived of the promotion of public hospital reform in their county. Approximately 59.24\% of the investigated medical staff members expressed that obvious changes occurred in their hospitals since the reform began in 2008 . Only $8.10 \%$ of the investigated medical staff members considered that the reform could significantly solve the main problems in public hospitals, and $68.32 \%$ considered that the reform could solve only part of the problems. Only $6.27 \%$ of the investigated medical staff thought that the reform could have evident effects that could solve the difficulty in the accessibility of medical service, and $71.52 \%$ thought that this reform could affect and alleviate this difficulty only to some extent. Only $7.25 \%$ of the investigated medical staff thought that the reform could 
Table 4 Analysis on the multiple factors influencing job satisfaction of medical staff in pilot county hospitals

\begin{tabular}{|c|c|c|c|c|c|}
\hline Medical staff in pilot county hospitals & Reference category & $B$ & $P$ & OR & $95 \% \mathrm{Cl}$ \\
\hline Age & 24 and below & & & & \\
\hline $25-34$ & & -0.231 & 0.522 & 0.794 & $(0.392-1.610)$ \\
\hline $35-44$ & & -0.549 & 0.279 & 0.577 & $(0.213-1.562)$ \\
\hline $45-54$ & & -0.603 & 0.309 & 0.547 & $(0.171-1.748)$ \\
\hline 55 and above & & -0.91 & 0.279 & 0.403 & $(0.077-2.093)$ \\
\hline Position & Medical technician & & & & \\
\hline Doctor & & -0.368 & 0.201 & 0.692 & $(0.394-1.216)$ \\
\hline Nurse & & -0.39 & 0.139 & 0.677 & $(0.404-1.136)$ \\
\hline Professional title & No title & & & & \\
\hline Junior title & & -0.249 & 0.719 & 0.779 & $(0.201-3.027)$ \\
\hline Middle title & & -0.458 & 0.506 & 0.633 & $(0.164-2.437)$ \\
\hline Senior title & & -0.417 & 0.555 & 0.659 & $(0.165-2.635)$ \\
\hline Years in professional working experience & $1-5$ years & & & & \\
\hline $6-10$ years & & 0.36 & 0.242 & 1.434 & $(0.784-2.623)$ \\
\hline $11-15$ years & & 0.72 & 0.054 & 2.055 & $(0.989-4.272)$ \\
\hline $16-20$ years & & 0.601 & 0.200 & 1.823 & $(0.728-4.567)$ \\
\hline 20 years and above & & 0.559 & 0.280 & 1.748 & $(0.635-4.817)$ \\
\hline Educational background & Technical secondary school and below & & & & \\
\hline Junior college & & 0.344 & 0.228 & 1.411 & $(0.806-2.469)$ \\
\hline Bachelor's degree & & 0.35 & 0.261 & 1.42 & $(0.771-2.615)$ \\
\hline Master's degree or above & & 0.352 & 0.464 & 1.421 & $(0.554-3.644)$ \\
\hline The number of hours spent at work everyday & $8 \mathrm{~h}$ and below & & & & \\
\hline $9 \mathrm{~h}$ & & -0.727 & $<0.001$ & 0.483 & $(0.327-0.713)$ \\
\hline $10 \mathrm{~h}$ & & -1.01 & $<0.001$ & 0.364 & $(0.230-0.576)$ \\
\hline $11 \mathrm{~h}$ and above & & -1.083 & $<0.001$ & 0.339 & $(0.202-0.568)$ \\
\hline The degree of work stress & Great pressure & & & & \\
\hline No pressure or moderate pressure & & 0.869 & $<0.001$ & 2.384 & $(1.666-3.411)$ \\
\hline Satisfaction on performance appraisal system & Dissatisfied & & & & \\
\hline Satisfied & & 0.808 & $<0.001$ & 2.243 & $(1.504-3.345)$ \\
\hline Satisfaction on concern showed by leaders & Dissatisfied & & & & \\
\hline Satisfied & & 0.302 & 0.206 & 1.352 & $(0.848-2.157)$ \\
\hline Satisfaction on hospital management & Dissatisfied & & & & \\
\hline Satisfied & & 0.715 & 0.009 & 2.043 & $(1.196-3.490)$ \\
\hline Satisfaction on compensation packages & Dissatisfied & & & & \\
\hline Satisfied & & 1.193 & $<0.001$ & 3.298 & $(2.410-4.513)$ \\
\hline Satisfaction on learning and training opportunities & Dissatisfied & & & & \\
\hline Satisfied & & 0.366 & 0.024 & 1.442 & $(1.050-1.980)$ \\
\hline
\end{tabular}

have evident effects that could solve the difficulty in the affordability of medical service, and $66.75 \%$ thought that this reform could only alleviate this difficulty to some extent.

The Pearson chi-square test method was used to assess the differences in the understanding and perception of the reform between medical staff in pilot county hospitals and medical staff in non-pilot county hospitals. The results showed that medical staff in pilot and non- pilot county hospitals exhibited significant differences in all the aspects aforementioned $(P<0.05$; Table 5$)$

\section{Discussion}

Medical staff members in pilot county hospitals were exposed to work-related stress to a higher extent

In this study, medical staff members in pilot county hospitals were exposed to work-related stress at a higher 
Table 5 The understanding and perception of public hospital reform of medical staff in pilot and non-pilot county hospitals

\begin{tabular}{|c|c|c|c|c|c|c|c|c|}
\hline \multirow[t]{2}{*}{ The understanding and perception of public hospital reform } & \multicolumn{2}{|c|}{$\begin{array}{l}\text { Pilot county } \\
\text { hospitals }(n=1531)\end{array}$} & \multicolumn{2}{|c|}{$\begin{array}{l}\text { Non-pilot county } \\
\text { hospitals }(n=737)\end{array}$} & \multicolumn{2}{|l|}{ Total } & \multirow[t]{2}{*}{$x^{2}$} & \multirow[t]{2}{*}{$P$} \\
\hline & $n$ & $\%$ & $n$ & $\%$ & $n$ & $\%$ & & \\
\hline \multicolumn{9}{|l|}{$\begin{array}{l}\text { The understanding degree of specific contents of the public hospital } \\
\text { reform programme }\end{array}$} \\
\hline Fully know & 70 & 4.57 & 14 & 1.90 & 84 & 3.70 & & \\
\hline Know a lot & 480 & 31.35 & 177 & 24.02 & 657 & 28.97 & & \\
\hline Know some & 484 & 31.61 & 304 & 41.25 & 788 & 34.74 & & \\
\hline Know a little & 424 & 27.69 & 201 & 27.27 & 625 & 27.56 & & \\
\hline Know nothing & 73 & 4.77 & 41 & 5.56 & 114 & 5.03 & 32.787 & $<0.001$ \\
\hline \multicolumn{9}{|l|}{ The perceived level of the reform promotion in this county } \\
\hline Perceive obviously & 770 & 50.29 & 293 & 39.76 & 1063 & 46.87 & & \\
\hline Perceive not obviously & 578 & 37.75 & 370 & 50.20 & 948 & 41.80 & & \\
\hline Perceive nothing & 46 & 3.00 & 22 & 2.99 & 68 & 3.00 & & \\
\hline Have no idea & 137 & 8.95 & 52 & 7.06 & 189 & 8.33 & 32.378 & $<0.001$ \\
\hline \multicolumn{9}{|l|}{ The perceived level of changes brought by reform since 2008} \\
\hline Change obviously & 907 & 59.24 & 231 & 31.34 & 1138 & 50.18 & & \\
\hline Change not obviously & 494 & 32.27 & 374 & 50.75 & 868 & 38.27 & & \\
\hline Change nothing & 65 & 4.25 & 100 & 13.57 & 165 & 7.28 & & \\
\hline Have no idea & 65 & 4.25 & 32 & 4.34 & 97 & 4.28 & 181.017 & $<0.001$ \\
\hline \multicolumn{9}{|l|}{$\begin{array}{l}\text { If public hospital reform could solve the problems faced by public } \\
\text { hospitals }\end{array}$} \\
\hline Solve the main problems & 124 & 8.10 & 38 & 5.16 & 162 & 7.14 & & \\
\hline Solve only part of the problems & 1046 & 68.32 & 491 & 66.62 & 1537 & 67.77 & & \\
\hline Solve nothing & 136 & 8.88 & 94 & 12.75 & 230 & 10.14 & & \\
\hline Have no idea & 225 & 14.70 & 114 & 15.47 & 339 & 14.95 & 13.797 & 0.003 \\
\hline \multicolumn{9}{|l|}{$\begin{array}{l}\text { If public hospital reform could solve the difficulty in accessibility of } \\
\text { medical service }\end{array}$} \\
\hline Have obvious effects & 96 & 6.27 & 27 & 3.66 & 123 & 5.42 & & \\
\hline Have some effects & 1095 & 71.52 & 469 & 63.64 & 1564 & 68.96 & & \\
\hline Have no effect & 175 & 11.43 & 132 & 17.91 & 307 & 13.54 & & \\
\hline Have no idea & 165 & 10.78 & 109 & 14.79 & 274 & 12.08 & 32.783 & $<0.001$ \\
\hline \multicolumn{9}{|l|}{$\begin{array}{l}\text { If public hospital reform could solve the difficulty in affordability of } \\
\text { medical service }\end{array}$} \\
\hline Have obvious effects & 111 & 7.25 & 24 & 3.26 & 135 & 5.95 & & \\
\hline Have some effects & 1022 & 66.75 & 428 & 58.07 & 1450 & 63.93 & & \\
\hline Have no effect & 226 & 14.76 & 175 & 23.74 & 401 & 17.68 & & \\
\hline Have no idea & 172 & 11.23 & 110 & 14.93 & 282 & 12.43 & 47.353 & $<0.001$ \\
\hline
\end{tabular}

extent than those in non-pilot county hospitals. This result could be attributed to the increasing number of patients, higher requirements for technical level and comprehensive quality of medical staff, and reform measures of hospital administration system caused by the public hospital reform [6]. Because of the health reform, the basic social health insurance system has been improved and medical cost and expenses have been reasonably controlled. The medical demand of the population is released and medical staff members would see more patients/day. In China, many jobs require employees to work for only $8 \mathrm{~h}$ a day, but the results of this study showed that more than half of the medical staff members $(51.86 \%)$ work for more than $8 \mathrm{~h}$ a day in pilot 
county hospitals. This finding showed that overtime working is common for medical staff in pilot county hospitals, which may increase the work stress.

\section{Pilot county hospitals have implemented some reform measures and got some positive effects, but there still are deficiencies}

Medical staff members in pilot county hospitals were more satisfied on current job, performance appraisal system, concern showed by leaders, hospital management, and compensation packages than those in non-pilot county hospitals. This finding suggested that the pilot county hospitals have implemented some reform measures to improve performance appraisal system and management system, to concern more on their staff, and to increase income and job satisfaction with this reform. These measures have showed some positive effects. However, there was no significant difference between satisfaction on learning and training opportunities of medical staff in pilot county hospitals than that in nonpilot county hospitals. This suggested that pilot county hospitals have not implemented sufficient effective measures to provide enough learning and training opportunities to medical staff.

\section{Medical staff members in pilot county hospitals have evidently less satisfaction on compensation packages and learning and training opportunities}

Within pilot county hospitals, medical staff members were evidently less satisfied with compensation packages and learning and training opportunities than with other work-related aspects. These indicated that medical staff members considered that current remuneration did not match the amount of work, and the learning and training opportunities currently provided for them did not satisfy the demands. At present, the average annual income of medical staff in county hospitals in China is basically the same as the average annual income of urban workers. But in many other counties, the income of medical staff is generally higher than that of other professions and is four or five times the income of urban workers. The income level of medical staff in county hospitals in China is well below the international situation. Hence, medical staff urgently needs improvement. In China, the income levels of medical staff in different county hospitals are basically the same. Therefore, dissatisfaction on current remuneration of medical staff in county hospital is a common problem.

\section{Significant influencing factors of job satisfaction of medical staff in pilot county hospitals have been determined}

Job satisfaction originates in the organizational psychology literature but has been adopted by some researchers in the field of human resources for health [7-9]. Locke defined this concept as "a pleasurable or positive emotional state resulting from the appraisal of one's job or job experiences" [10]. In the field of health care, job satisfaction of medical staff determines the quality of service delivery to patients [11,12]. Poor job satisfaction is associated with absenteeism, employee turnover in an organization, and eventual exhaustion [13,14].

To assess job satisfaction, we further analysed the factors influencing the job satisfaction of medical staff in pilot county hospitals by univariate and multivariate analyses. Univariate analysis results showed that sociodemographic characteristics (including age, educational background, position, professional title, and years in professional working experience), working situation (including the number of hours spent at work every day and work stress), and satisfaction on some work-related factors (including satisfaction on performance appraisal system, concern showed by leaders, hospital management, compensation packages, and learning and training opportunities) were related to the job satisfaction of medical staff in pilot county hospitals.

However, multivariate analysis results showed that only the number of hours spent at work, work stress, and satisfaction on performance appraisal system, hospital management, compensation packages, and learning and training opportunities were significantly associated with job satisfaction of medical staff in pilot county hospitals. None of the sociodemographic characteristics exhibited a significant correlation. It indicated that the influence on job satisfaction of medical staff caused by sociodemographic characteristics in this study was weaker than that caused by working situation and satisfaction on work-related factors. This phenomenon could be attributed to great changes in the working situations of medical staff and work-related factors in pilot county hospitals in China as a result of the implementation of the health-care reform and hospital reform. Therefore, sociodemographic characteristics did not strongly influence job satisfaction, whereas working situations and satisfaction on work-related factors were statistically significant.

The results of multivariate analysis indicated long hours spent at work each day and high work-related stress encountered could result in less job satisfaction. By comparison, a high satisfaction on performance appraisal system, hospital management, compensation packages, and learning and training opportunities could indicate high job satisfaction. These findings are consistent with those in earlier studies on job satisfaction [14-28]. For example, health workers in Ghana overwhelmingly identify low salaries as the main source of dissatisfaction on an interviewer-administered questionnaire [29]. Kumar and co-workers found that factors influencing the satisfaction level include low salaries, lack 
of training opportunities, improper supervision, and inadequate financial rewards. Marinucci and co-workers conducted a survey in seven sub-Saharan African countries [30], and the result shows that professional development and training opportunities are the most important factor resulting in job satisfaction as indicated by approximately 90\% of the total interviewees. Peters and his co-workers conducted a survey in India and found that many employees rate "training opportunities" as one of the motivating factors [31]. A review of 12 empirical studies on the motivation of developing and developed countries has found that seven major job characteristics are important determinants of job motivation, including opportunities for personal development, pay/rewards, management practices, and organizational policies [32]. A study in Vietnam has also found that the main motivating factors of health workers include the following: the appreciation expressed by their managers, competitive income, and training [33].

\section{Suggestions for improving job satisfaction of medical staff in pilot county hospitals}

Therefore, the government officials and hospital administrators should pay attention to these influencing factors and focus on the demands of medical staff in pilot county hospitals. What should be done most at present mainly include three aspects [34,35]. 1) The hospital administrators should have more concern for the working situation of medical staff in pilot county hospitals, properly assign and arrange work, and appropriately reduce workload and work stress to promote job satisfaction and active participation of medical staff in pilot county hospitals. 2) The government officials and hospital administrators should improve the system of compensation packages, promote income levels, and make the income match the workload and technical value of medical staff in pilot county hospitals. 3) More learning and training opportunities should be provided and created to medical staff in pilot county hospitals, in order to help them to improve their professional level and meet their individual development requirements. 4) More physical and mental health considerations and better performance appraisal system and management system should be provided for medical staff in pilot county hospitals, in order to promote their job satisfaction. 5) In a previous study, the participation of medical staff in decision-making significantly affected job satisfaction [28]; as such, democratic management can be applied in pilot county hospitals.

\section{Medical staff in pilot county hospitals exhibited better understanding of the public hospital reform programme and more positive attitude towards it, but it still needs improvement}

The results of the data analysis showed that the medical staff in pilot county hospitals exhibited a better understanding of the specific contents of the public hospital reform programme, more optimistic perception of the changes caused by the reform, and more firm confidence and positive attitude towards the reform compared with the medical staff in non-pilot county hospitals. These findings indicated that the reform measures implemented in pilot county hospitals have resulted in some positive effect, and the medical staff in pilot county hospitals experienced more advantages from this reform. However, there still were some medical staff members in pilot county hospitals who showed insufficient understanding and perception of the reform and hold negative attitude towards the effect of the reform. These findings suggested that the understanding, perception, and attitude towards public hospital reform of the medical staff in pilot county hospitals still need improvement. Therefore, 1) the government should provide more implementing rules of the reform policy, to make the reform policy more clearly and operable; 2) the government officials and hospital administrators can guide medical staff members with different individual characters by using different methods to learn the knowledge and importance of this reform in pilot county hospitals; 3) to promote the active implementation of public hospital reform, government officials and hospital administrators should guide and encourage the medical staff in pilot county hospitals to take part in the reform, and make more publicity on the benefits of the reform utilizing multiple forms, such as conferences, posters, and TV shows.

\section{Possible limitations}

In this study, there were three possible limitations: first, the cross-sectional design with job satisfaction. It was difficult to establish a causal conclusion, and the longitudinal survey might be carried out to confirm the causal conclusion in our future study. Second, the measurements were performed by a self-administrated method. Then, it is possible that the respondents might have overreported or underreported their level of job satisfaction and satisfaction on work-related factors and understanding and perception of the reform. Third, given that the study was conducted only in counties of Hubei province, the findings of the study may or may not be generalized to medical staff working in other areas in China.

\section{Conclusions}

The results in this study indicated that pilot county hospitals have implemented some measures to improve the performance appraisal system and management system, provide adequate care for their medical staff, and increase medical staff's income and job satisfaction through the reform. Pilot county hospitals have experienced some positive effects but there still are deficiencies. Within pilot county hospitals, work stress increased and less than half of the medical staff members were 
very satisfied or satisfied with current job. To promote the job satisfaction of medical staff in pilot county hospitals, the government officials and hospital administrators should pay attention to these influencing factors of job satisfaction and focus on the reasonable demands of medical staff in pilot county hospitals.

In addition, the medical staff members in pilot county hospitals exhibited a better understanding of the public hospital reform programme and showed more firm confidence and positive attitude towards the reform than the medical staff members in non-pilot county hospitals. These findings indicated that the reform measures implemented in pilot county hospitals have resulted in some positive effect, and the medical staff in pilot county hospitals experienced more advantages from this reform. However, there still were some medical staff members in pilot county hospitals who showed insufficient understanding and perception of the reform and hold negative attitude towards the effect of the reform. These findings suggested that the understanding, perception, and attitude towards the public hospital reform of the medical staff and the publicity and education of the reform in pilot county hospitals still need improvement.

\section{Competing interests}

The authors declare that they have no competing interests.

\section{Authors' contributions \\ $\mathrm{PF}, \mathrm{ZL}$, and $\mathrm{ZF}$ participated in the literature search and the design of the study and took part in the survey and the data analysis. PF and ZL contributed to the data interpretation and the writing of the article. All authors have read and approved the final version.}

\section{Authors' information}

PF is a professor, a Ph.D. candidate superior, the Deputy Dean of the Schoo of Health and Medicine Management, and the Director of the Hospital Management and Development Research Center, Tongji Medical College, Huazhong University of Science and Technology. He obtained his Ph.D. in Demography at the Institute of Population Research, Peking University, Beijing, People's Republic of China, in 1999-2002. He was a post-doctoral fellow in the Center for Health Policy, Stanford University, U.S.A., in 2003-2004. Research areas include hospital management, population and health, and health resource management.

$\mathrm{ZL}$ is a Ph.D. lecturer at the School of Health Management, Guangzhou Medical University. Research interests include hospital management, health resource management, and health policy research.

ZF is a Master's degree candidate at The London School of Economics and Political Science. Research interests include strategic management and hospital management.

\section{Acknowledgements}

This research was supported by the National Natural Science Foundation of China (No. 71073062). We also thank Australian health policy expert Dr Chris Scarf and Mrs Ana Scarf for their help with this article.

\section{Author details}

${ }^{1}$ School of Health and Medicine Management, Tongji Medical College, Huazhong University of Science and Technology, 13 Hangkong Road, Qiaokou District, Wuhan 430030, China. ${ }^{2}$ School of Health Management, Guangzhou Medical University, 195 Dongfeng West Road, Yuexiu District, Guangzhou 510182, China. ${ }^{3}$ The London School of Economics and Political Science, 13420, Houghton Street, London WC2A 2AE, UK.
Received: 23 April 2013 Accepted: 29 April 2015

Published online: 16 May 2015

\section{References}

1. Liu Y. Reforming China's health care: for the people, by the people? Lancet. 2009;373:281-3.

2. Li L. The challenges of healthcare reforms in China. Public Health. 2011;125:6-8.

3. Zhu C. Launch of the health-care reform plan in China. Lancet. 2009;373:1322-4.

4. National Health and Family Planning Commission of the People's Republic of China. National plan for rural health system construction and development. 2006. http://www.nhfpc.gov.cn/zhuzhan/wsbmgz/201304/965d43777b6e4a2993 ff139c08ce534d.shtml. (in Chinese) Accessed 6 May 2015.

5. Ding H, Sun X, Chang WW, Zhang L, Xu XP. A comparison of job satisfaction community health workers before and after local comprehensive medical care reform: a typical field investigation in Central China. PLoS One. 2013;8, e73438.

6. Hou JL, Li N, Lu L, Li JZ, Ma J. Research on job satisfaction degree primary healthcare workers after health care system reform in Shanghai City. Chin Hospital Manage. 2013;33:10-2.

7. Tzeng $H$. The influence of nurses' working motivation and job satisfaction on intention to quit an empirical investigation in Taiwan. Int J Nurs Stud. 2002;39:867-78.

8. Pillay R. Work satisfaction of professional nurses in South Africa: a comparative analysis of the public and private sectors. Hum Resour Heal. 2009;7:15.

9. Sibbald B, Enzer I, Cooper C, Rout U, Sutherland V. GP job satisfaction in1990 and 1998: lessons for the future? Fam Pract. 1987;2000(17):364-71.

10. Locke $E$. The nature and causes of job satisfaction. In: Dunnette M, Hough L, editors. The handbook of industrial and organizational psychology. Palo Alto, CA: Consulting Psychologists Press; 1976. p. 1319-28.

11. Haas JS, Cook EF, Puopolo AL, Burstin HR, Cleary PD, Brennan TA. Is the professional satisfaction of general internists associated with patient satisfaction? J Gen Intern Med. 2000;15:122-8.

12. Bodur S. Job satisfaction of health care staff employed at health centres in Turkey. Occup Med (Lond). 2002;52:353-5.

13. McManus IC, Keeling A, Paice E. Stress, burnout and doctors' attitudes to work are determined by personality and learning style: a twelve year longitudinal study of UK medical graduates. BMC Med. 2004;2:29.

14. Visser MR, Smets EM, Oort FJ, De Haes HC. Stress, satisfaction and burnout among Dutch medical specialists. CMAJ. 2003;168:271-5.

15. Zhang YM, Feng XS. The relationship between job satisfaction, burnout, and turnover intention among physicians from urban state-owned medical institutions in Hubei, China: a cross-sectional study. BMC Health Serv Res. 2011;11:235-48

16. Hills D, Joyce C, Humphreys J. Validation of a job satisfaction scale in the Australian clinical medical workforce. Eval Health Prof. 2011:35:47-76.

17. Nylenna M, Gulbrandsen P, Førde R, Aasland OG. Unhappy doctors? A longitudinal study of life and job satisfaction among Norwegian doctors 1994-2002. BMC Health Serv Res. 2005;5:44.

18. Pathman DE, Konrad TR, Williams ES, Scheckler WE, Linzer M, Douglas J. Career Satisfaction Study Group. Physician job satisfaction, dissatisfaction, and turnover. J Fam Pract. 2002:51:593.

19. Murrells T, Robinson S, Griffiths P. Is satisfaction a direct predictor of nursing turnover? Modelling the relationship between satisfaction, expressed intention and behaviour in a longitudinal cohort study. Hum Resour Heal. 2008;6:22.

20. Lambrou P, Kontodimopoulos N, Niakas D. Motivation and job satisfaction among medical and nursing staff in a Cyprus public general hospital. Hum Resour Heal. 2010;8:26-34.

21. McGlynn K, Griffin MQ, Donahue M, Fitzpatrick JJ. Registered nurse job satisfaction and satisfaction with the professional practice model. J Nurs Manage. 2012;20:260-5.

22. Pillay R. Work satisfaction of medical doctors in the South African private health sector. J Health Organ Manag. 2008;22:254-68.

23. Taylor K, Lambert T, Goldacre M. Career destinations, job satisfaction and views of the UK medical qualifiers of 1977. J R Soc Med. 2008;101:191-200.

24. Wenderlein FU. Work satisfaction and absenteeism of nursing staff-comparative study of 1021 nurse trainees and nurses. Gesundheitswesen. 2003;65:620-8.

25. Sara DG, Joeri H, Roland P. Revisiting the impact of job satisfaction and organizational commitment on nurse turnover intention: an individual differences analysis. Int J Nurs Stud. 2011:48:1562-9.

26. Gardulf A, Orton ML, Eriksson LE, Undén M, Arnetz B, Kajermo KN, et al. Factors of importance for work satisfaction among nurses in a university hospital in Sweden. Scand J Caring Sci. 2008;22:151-60. 
27. Tellez M. Work satisfaction among California registered nurses: a longitudinal comparative analysis. Nurs Econ. 2012;30:73-81.

28. Janus K, Amelung VE, Baker LC, Gaitanides M, Schwartz FW, Rundall TG. Job satisfaction and motivation among physicians in academic medical centers: insights from a cross-national study. J Health Polit Policy Law. 2008;33:1133-67.

29. Agyepong I, Anafi P, Asiamah E, Ansah EK, Ashon DA, Narh-Dometey C. Health worker (internal customer) satisfaction and motivation in the public sector in Ghana. Int J Health Plann Manage. 2004;19:319-36.

30. Kumar R, Ahmed J, Shaikh BT, Hafeez R, Hafeez A. Job satisfaction among public health professionals working in public sector: a cross sectional study from Pakistan. Hum Resour Health. 2013;11:2

31. Peters DH, Subrata C, Prasanta M. Job satisfaction and motivation of health work in public and private sectors: cross-sectional analysis from two Indian states. Laura Steinhardt Hum Resour Health. 2010;8:27.

32. Dolea C, Adams O. Motivation of health care workers-review of theories and empirical evidence. Cah Sociol Demogr Med. 2005;45:135-6.

33. Dieleman M, Cuong PV, Anh LV, Martineau T. Identifying factors for job motivation of rural health workers in North Viet Nam. Hum Resour Health. 2003;5:10.

34. Luo Z, Bai X, Min R, Tang C, Fang P. Factors influencing the work passion of Chinese community health service workers: an investigation in five provinces. BMC Fam Pract. 2014;15:77.

35. Sun $Y, L u o Z$, Fang $P$. Factors influencing the turnover intention of Chinese community health service workers based on the investigation results of five provinces. J Community Health. 2013;38:1058-66.

\section{Submit your next manuscript to BioMed Central and take full advantage of:}

- Convenient online submission

- Thorough peer review

- No space constraints or color figure charges

- Immediate publication on acceptance

- Inclusion in PubMed, CAS, Scopus and Google Scholar

- Research which is freely available for redistribution 